\title{
The watched pot does boil: A case of the wrong control group
}

\author{
DANIEL S. LORDAHL and SAMUEL BERKOWITZ \\ The Florida State University, Tallahasee, Florida 32306
}

\begin{abstract}
The question posed was whether or not the attention to a forthcoming event (expectancy) changes the subjective passage of time as compared with a standard control condition in which the passage of time is covertly monitored. Each of 36 subjects served under six experimental conditions, involving either expectancy or control intervals and three different objective durations. The data showed no significant effect of expectancy, per se. An interpretation of the watched pot phenomenon was offered.
\end{abstract}

There is no doubt in the minds of most people that the watched pot phenomenon is real. Even a young child waiting the last few days until Christmas or some other special day will verify that the passage of time is much slower than "usual." However, the theoretical analysis of such an effect is not quite as simple as it may appear. It is well known that time judgments are quite different during intervals filled with activities (Fraisse, 1963; Shectman, 1970), as compared with judgments from intervals during which a person is attending to the passage of time. The magnitude of the underestimation of time during intervals in which information processing is taking place may be quite large. For example, Lordahl (1973) found under some conditions that the mean estimate of filled intervals was 33\% less than the mean for unfilled intervals, which were, in turn, about equal to the objective lengths. Also, Curton and Lordahl (in press) found estimates from filled intervals which averaged $45 \%$ less than control values. Thus, it could be argued that the watched pot effect is phenomenologically impressive because one compares an interval during which attention to the passage of time is taking place with the more usual interval, in which much cognitive activity is present.

But, it is also possible that there may be true differences between two kinds of "unfilled" intervals, those in which the passage of time is simply being monitored and those in which attention is directed toward the occurrence of a future event. Let us refer to the latter as an expectancy situation. A study by Loehlin (1959) and other work summarized in Orme (1969) suggest that there could be a difference in the subjective passage of time between the ordinary control condition in which subjects attempt to time an interval and the expectancy situation.

The purpose of the present study was to compare time estimation of intervals during which subjects are

This paper is sponsored by George Weaver, who takes full editorial responsibility for its contents. Requests for reprints should be sent to Daniel S. Lordahl, Department of Psychology, The Florida State University, Tallahassee, Florida 32306.

The participation of the second author was funded, in part, by Grant GW-8127 from the National Science Foundation. waiting for an expected event to take place with estimations from control conditions, i.e., those in which the duration of an interval is attended to by subjects.

\section{METHOD}

\section{Subjects}

The data from 36 psychology students, serving as subjects for course credit, were used. Data from another four subjects were dropped on account of procedural errors.

\section{Task and Materials}

Two basic types of time estimation trials were used. First, on some trials subjects were given oral "start" and "stop" commands and were required to attempt to time the intervals by silently counting off the number of seconds. This standard empty interval procedure will be referred to as control trials. The second type of estimation trial involved the use of a die and a plastic drinking glass. When so instructed, subject picked up the glass with the die contained within and waited for the command "roll." At this point, the subject turned the glass upside down on the table and gave an estimate of the length of time he had held the container and die. On each such expectancy trial, subject had the opportunity of winning $\$ 0.25$ if the outcome on the die matched one of two numbers chosen by the experimenter before the trial. Although the outcomes of such trials were rigged by the experimenter, the subjects were led to believe that they had a probability of $1 / 3$ of winning $\$ 0.25$ on each such expectancy trial. The plastic glass was opaque so that on such trials subjects gave their time estimates prior to observing the outcome of the die toss. The winning numbers were shown on cards to the subject after the die toss and time estimate.

\section{Design and Procedures}

Two preliminary trials, described below, were followed by six experimental trials for each subject. These latter trials were composed of one each of the combinations of a 2 by 3 orthogonal design where the first factor was the type of trial, either control or expectancy, and the second factor was the length of the time interval, with the levels being 15,40 , and $120 \mathrm{sec}$. These six trials were ordered in a Latin square arrangement. In addition, this basic Latin square was used under three levels of another variable, the particular expectancy trial which was rewarded. For example, all subjects assigned to the first square were rewarded only on the first of the three expectancy trials, all subjects in the second square were rewarded only on the second expectancy trial, etc. In each sequence of each use of the square, two subjects were assigned, yielding the total of 36 subjects in the study.

The two preliminary trials may be termed (a) an unexpected 
time estimation trial and (b) a rewarded expectancy practice trial. First, the two subjects assigned to any given sequence of the basic design were treated differently on the first preliminary trial. One of them was simply asked to wait a moment (after general instructions had been given) while the experimenter went to get his stopwatch from the next room. The other type of subject had already been given the expectancy trial instructions and was holding the die container when the experimenter left the room. In each case, the experimenter returned $30 \mathrm{sec}$ after closing the door and asked each subject for an estimate of the interval between the closing and opening of the lab room door. All subjects receiving the expectancy trial first failed to win the game.

On the second preliminary trial, all subjects were given the expectancy condition with a 10-sec interval. In addition, in order to increase credibility, all subjects won and were given their quarter on this trial.

The instructions to subjects stressed attention to the forthcoming roll of the die on expectancy trial and specifically asked subjects not to try to time the delay interval on such trials.

No feedback as to true durations was given during the experimental session, and subjects were asked not to disclose the nature of the experiment to other students.

The intertrial intervals were held as close as practicable to $30 \mathrm{sec}$, and the session was completed in less than $30 \mathrm{~min}$.

\section{RESULTS}

All time estimates (in sec) were converted to a rate of internal clock, RIC, index by dividing them by the true duration of the interval. Thus, all RIC values less than unity indicate that the corresponding time estimates were less than the true interval, while RIC values above unity indicate overestimation by the subject.

The RIC values from the six experimental trials were subjected to an analysis of variance appropriate to the Latin square design employed. None of the effects, including various interactions, was significant. ${ }^{1}$ The overall mean RIC was 1.023 , which is not reliably different than unity. In addition, the mean difference in RIC between expectancy trials and control trials was .055 , and $95 \%$ confidence interval for this mean difference, $d$, was $-.044 \leqslant d \leqslant .154$. The $F(1,18)=1.36$ for this variable, with $p>.20$. These results certainly suggest no strong expectancy effect. Moreover, even though the Type of Trial by Duration interaction was not significant in the analysis, the difference between control and expectancy type trials was examined separately at each of the three durations. Again no significant effects of expectancy were detected.

The two preliminary trials may be treated as a simple two condition experiment, where half the subjects received an expectancy condition on Trial 1 and the others a control condition. The mean RIC was .68 for the control condition on Trial 1 and was .86 for the expectancy conditions. These means do not differ reliably, $t(34)=1.10, p>.20$. However, on the second preliminary trial, the common expectancy trial on which all subjects won, the mean difference between these two groups of subjects was significant. The mean for the subjects who had received a control condition on Trial 1 was .99 and the mean for the other subjects was 1.40 , $t(34)=2.29, p<.05$

\section{DISCUSSION}

There was no suggestion that the expectation of a future event and attention to such a forthcoming event has any appreciable effect on time estimation. The comparision of this expectation condition was an unfilled interval, in which subjects covertly kept track of time.

The difference between average RIC values on the second preliminary trial, which was an expectancy trial for all subjects, was probably due to an arousal effect of failure. The reader should recall that the subjects given an expectancy-type trial on Trial 1 were uniformly unreinforced. It is suggested that this treatment was sufficient to arouse these subjects briefly, resulting in higher time estimates on the next preliminary trial.

It must be emphasized that failure of the expectancy variable to affect time estimates in this study should not be generalized too broadly. First, the rewards used here to produce "expectancy" were not large. Secondly, the longest duration employed was $120 \mathrm{sec}$. Although this period may seem long subjectively in a laboratory setting, there can be no doubt that intervals of hours or days are quite a different situation.

In a more positive manner, it should be pointed out that the intervals used here are similar to those appropriate to the colloquial watched pot phenomenon. The conclusion drawn by the authors is that the subjective impression of a slowing of the passage of time under expectancy conditions is not a function of the expectancy itself, but is due to the comparision of such an experience with similar intervals which have been filled with cognitive activities more complex than those of simple time keeping. In other words, the subjective passage of time under usual day-to-day conditions for an active human is faster than under unfilled interval conditions in which subjects concentrate on the passage of time.

\section{REFERENCES}

Curton, E. D., \& Lordahl, D. S. The effects of attentional focus and arousal on time estimation. Journal of Experimental Psychology, in press.

Fraisse, P. The psychology of time. New York: Harper \& Row, 1963.

Loehlim, J. C. The influence of different activities on the apparent length of time. Psychological Monographs, 1959, 73, (4, Whole No. 474).

Lordahl, D. S. Effects of processing external and internal information on time estimation. Paper presented at the meeting of the Southeastern Psychological Association, New Orleans, 1973.

Orme, J. E. Time, experience and behavior. New York: American Elseview Publishing Company, 1966.

Shectman, F. Time estimation, sequence effects and filling activities. Perceptual and Motor Skills, 1970, 30, 23-26.

\section{NOTE} 1.92 .

1. All p-values were greater than .10 . The largest $F(2,36)=$ (Received for publication October $7,1974$. 